\title{
Multi-modal correlative chemical imaging of aquatic microorganisms
}

Vivian Merk $^{1}$, Johan Decelle ${ }^{2}$, Si Chen $^{3}$ and Derk Joester ${ }^{4}$

${ }^{1}$ Florida Atlantic University, Boca Raton, Florida, United States, ${ }^{2}$ University of Grenoble Alpes, Grenoble, Auvergne, France, ${ }^{3}$ Argonne National Laboratory, Lemont, Illinois, United States,

${ }^{4}$ Northwestern University, Illinois, United States

Multi-scale chemical imaging tools are critical to investigate cellular architecture and organization, ion transmembrane transport and trace element homeostasis of complex biological systems. It is known that an organism's inorganic composition dynamically changes in response to physiological stimuli, genetic makeup, and developmental state. This research explores how aquatic microorganisms mineralize trace elements from the ambient environment, resulting in characteristic cellular element stoichiometries.

A paramount function of biological membranes is their ability to select between chemically similar ions. Living organisms from various kingdoms achieve remarkable selectivity in the accumulation and immobilization of metal ions [1]. A prime example of ultraselective ion accumulation in nature can be found in the marine zooplankton Acantharia, which build their entire skeletons from strontium sulfate despite comparably low concentrations of strontium in seawater [2]. Eukaryotic organisms, such as Acantharea, play a key role in oceanic biogeochemistry, particularly in the global strontium-barium [3] and carbon-nitrogen cycle [4]. Intriguingly, some species of Acantharia harbor endosymbiotic microalgae in the endoplasm, lending them indirect photosynthetic activity [4,5]. Due to the wide distribution of Acantharia across the world' oceans, interactions between heterotrophic protist and microalgae symbiont are an integral part of marine ecosystems. To probe the elemental distribution with sub-100nm resolution, we combined hard X-ray fluorescence with differential phase-contrast imaging at the 9-ID-B endstation (Xradia Bionanoprobe) of the Advanced Photon Source. The sample preparation workflow included highpressure freezing, freeze substitution, resin embedding, and thin sectioning. Metalloproteins or enzymes are associated with specific cell organelles, including the capsular membrane, nucleus, nucleolus, and calcium-storing organelles, and can be identified based on their distinct elemental composition (Figure 1). The nuclei of endosymbiotic microalgae Phaeocystis, for instance, contain hotspots of phosphorous and iron (Figure 2). Algal chloroplasts are enriched in iron, copper and zinc, which is consistent with photosynthetic activity (Figure 2).

Cyanobacteria, also called blue-green algae, are an ancient group of prokaryotic microbes. In abundance, these aquatic microorganisms can produce harmful algal blooms that impair human health and aquatic ecosystems [6]. This study seeks to quantify the effect of micronutrients on the growth of the freshwater cyanobacteria Microcystis aeruginosa, their photosynthetic activity and cyanotoxin production. Towards this goal, X-ray fluorescence imaging was used to quantitatively image the trace element distribution in frozen-hydrated cells. The cryogenic capabilities at beamline 9-ID-B are suitable for visualizing the ultrastructure of cultured and field samples in their near-native state, thereby avoiding the invasive process of embedding. Following a multimodal and correlative imaging approach, we combined X-ray microscopy with complementary chemical imaging techniques that use different physical principles, such as liquidphase Atomic Force Microscopy, Raman or Electron Microscopy. Combining multiple imaging modalities at the (sub)cellular level harbors tremendous scientific potential for marine biology, biomineralization, environmental science, and geobiochemistry. 


\section{Acknowledgment statement}

This work was funded through the National Science Foundation (DMR-1508399) and the Swiss National Science Foundation (SNSF). J. D. is supported by the LabEx GRAL (ANR-10-LABX-49-01) and Pôle CBS from the University of Grenoble Alpes. This research used resources of the Advanced Photon Source, a U.S. Department of Energy (DOE) Office of Science User Facility operated for the DOE Office of Science by Argonne National Laboratory under Contract No. DE-AC02-06CH11357.

Figure 1. Synchrotron XRF micrograph of marine Acantharian collected with Bionanoprobe (Advanced
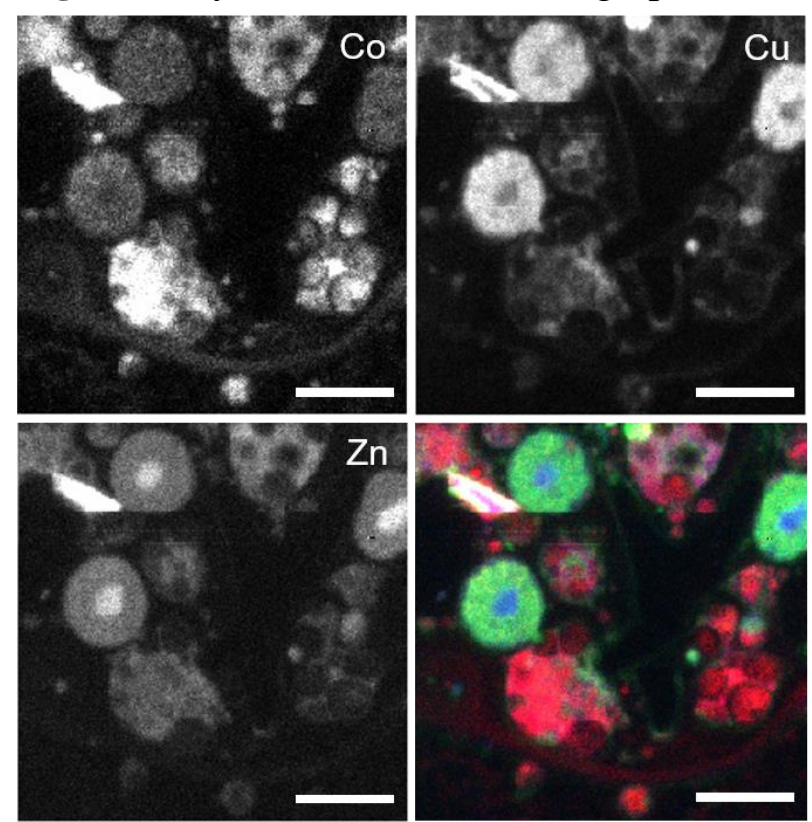

Photon Source). Elemental mapping shows high concentrations of copper in nuclei, zinc in nucleoli and cobalt in capsular membrane. Scale bar corresponds to $5 \mu \mathrm{m}$.
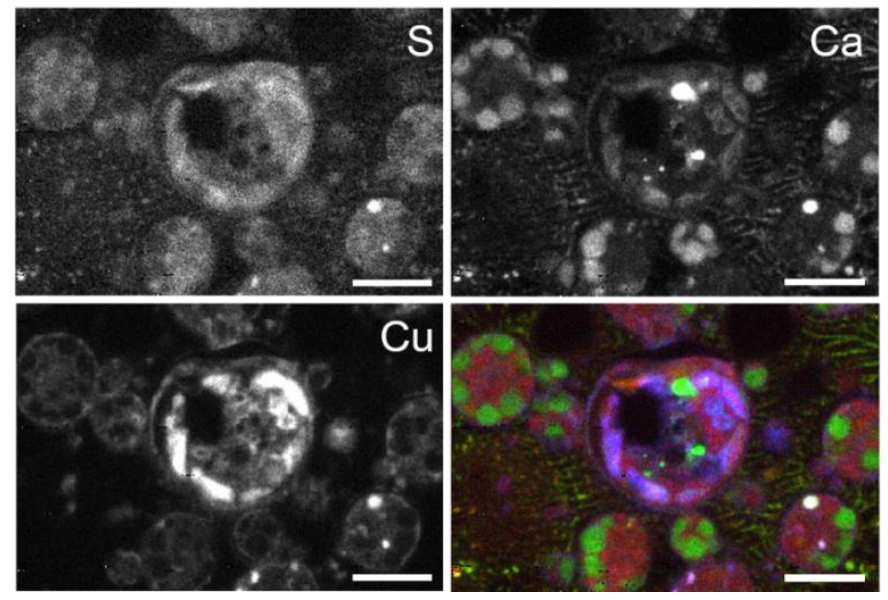

Figure 2. Synchrotron XRF micrograph of marine Acantharian collected with Bionanoprobe (Advanced Photon Source). Elemental mapping from zooplanktonic organism and microsymbiont Phaeocystis. The algal nuclei are enriched in phosphorous and iron, whereas thylakoids contain high amounts of copper and zinc. Scale bar corresponds to $5 \mu \mathrm{m}$. 


\section{References}

[1] M. Krejci, B. Wasserman, L. Finney, I. McNulty, D. Legnini, S. Vogt, D. Joester, Selectivity in biomineralization of barium and strontium. Journal of Structural Biology, 2011. 176(2): p. 192-202.

[2] V. Merk, J. Decelle, S. Chen, A. Lanzirotti, M. Newville, O. Antipova, D. Joester, Selective Ion Accumulation in Biomineralizing Marine Acantharia, Microscopy and Microanalysis, 2019, 25 (S2): p. 1072-1073.

[3] R. Bernstein, R. Byrne, P. Betzer, A. Greco, Morphologies and transformations of celestite in seawater: The role of acantharians in strontium and barium geochemistry. Geochimica et Cosmochimica Acta, 1992, 56(8): p. 3273-3279.

[4] J. Decelle, I. Probert, L. Bittner, Y. Desdevises, S. Colin, C. de Vargas, M. Galí, R. Simó, F. Not, An original mode of symbiosis in open ocean plankton. Proceedings of the National Academy of Sciences, 2012. 109(44): p. 18000-18005.

[5] J. Decelle, H.Stryhanyuk, B. Gallet, G. Veronesi, M. Schmidt, S. Balzano, S. Marro, C. Uwizeye, P. Jouneau, J. Lupette, Algal remodeling in a ubiquitous planktonic photosymbiosis. Curr. Biol. 29, 968-978 (2019).

[6] M. Harke, M. Steffen, C, Gobler, T. Otten, S. Wilhelm, S. Wood, H. Paerl, A review of the global ecology, genomics, and biogeography of the toxic cyanobacterium, Microcystis spp. Harmful Algae 54, 4-20 (2016). 